\title{
Data to the Hymenoptera fauna of Sicily (Hymenoptera: Symphyta and Aculeata)
}

\author{
ATtila HARIs ${ }^{1} \&$ Zsolt JóZAN ${ }^{2}$ \\ ${ }^{1} \mathrm{H}-1076$ Budapest, Garay utca 19 2/20, Hungary \\ e-mail: attilaharis@yahoo.com \\ ${ }^{2} \mathrm{H}-7543$ Mernye, Rákóczi utca 5, Hungary \\ e-mail: jozan.zsolt@citromail.hu
}

Haris, A. \& Józan, Zs.: Data to the Hymenoptera fauna of Sicily (Hymenoptera: Symphyta and Aculeata). Abstract: Results of Hymenoptera collections in Sicily are published. 11 Symphyta and 100 Aculeata species are listed mainly from the Nebrodi Mountains and from the Capo di Milazzo. Empria liturata (Gmelin, 1790) and Nomada glaucopis Pérez, 1884 are new records for Sicily.

Keywords: Hymenoptera, Symphyta, Aculeta, fauna, Sicily, new record.

\section{Introduction}

In 2013, the first author, A. Haris spent 25 days to investigate the Hymenoptera fauna of Sicily. Although Aculeata and Symphyta fauna is well researched and the local faunistic lists are completed (Liston et al. 2013, NobiLe 1988, 1989, 1990, 1995a, b, Nobile \& Campadelli 1998, Nobile et al. 2005, Nobile \& Tomarchio 1998, Nobile \& Turrisi 1997, 1999, 2004, 2015, Pagliano 1985, 1986, 1988a, b, c, 1990, 1992, 1993, 1994, Pagliano \& Nobile 1993, 1995, Pagliano \& Strumia 2007, Pesarini \& Turrisi 2001, Soika 1944, Soika \& Borsato 1995, Strumia 2015, Tomarchio \& Turrisi 2006, TurRisi 1999, b, c, 2009, 2011, TuRRISI \& OLMi 2009, Vicidomini 2007, Vicidomini et al. 2005) the investigation of separate regions of Sicily is still important task for the future.

In this paper, we provide Hymenotera faunistic data for the Nebrodi Mountains and for the Milazzo Peninsula.

\section{Material and methods}

The material was collected by A. Haris in 2013. Four locations was investigated. $90 \%$ of the specimens were collected from the Nebrodi Mountains (Figs. 1 and 4) between San Fratello and Cesaro and from the Capo di Milazzo (Figs. 2, 3 and 5). Few species are collected from the Rocca di Cefalu (Fig. 6) and from Etna (Fig. 7). 
Attila Haris identified the Symphyta part of the collection and Zsolt Józan the Aculeta part with help of Fabrizio Turrisi, Vittorio Nobile and Andreas Müller.

The presence of Empria liturata (GMELIN, 1790) is confirmed by genitalia dissection.

The voucher specimens are deposited at the Nature History Department of the RipplRónai Museum, Kaposvár, (Hungary).

\section{List of sampling places with coordinates and collecting dates}

1. Patti, N $38^{\circ} 07.288^{\prime}$ E $15^{\circ} 01.510^{\prime}, 472$ m. 20. 04. 2013

2. Braidi, N $38^{\circ} 04.904^{\prime} \mathrm{E} 15^{\circ} 00.560^{\prime}, 587 \mathrm{~m}$. 20. 04. 2013

3. Blaida, no coordinata avilable, 20. 04. 2013

4. San Fratello, N $37^{\circ} 58.893^{\prime}$ E $14^{\circ} 36.784^{\prime}, 808$ m. 27. 04. 2013

5. San Fratello, N $37^{\circ} 57.239^{\prime}$ E $14^{\circ} 37.324^{\prime}, 1037$ m. 27. 04.2013

6. San Fratello, N $37^{\circ} 56.572^{\prime}$ E $14^{\circ} 37.555^{\prime}, 1380$ m. 27. 04.2013

7. San Fratello, N $37^{\circ} 55.861^{\prime}$ E $14^{\circ} 38.314^{\prime}$ ', 1374 m. 27. 04. 2013

8. Acquedolci, N $38^{\circ} 04.926^{\prime} \mathrm{E} 14^{\circ} 36.309^{\prime}, 27.04 .2013$,

9. San Fratello, N $37^{\circ} 58.897^{\prime}$ E $14^{\circ} 36.782^{\prime}, 802$ m. 01. 05. 2013

10. San Fratello, N $37^{\circ} 58.411^{\prime}$ E $14^{\circ} 37.043^{\prime}, 871$ m. 01. 05. 2013

11. San Fratello, N $37^{\circ} 57.301^{\prime}$ E $14^{\circ} 37.323^{\prime}, 1010 \mathrm{~m} \mathrm{01.05.} 2013$

12. San Fratello, N $37^{\circ} 56.561^{\prime} \mathrm{E} 14^{\circ} 37.559^{\prime}, 1168 \mathrm{~m} \mathrm{01.05.} 2013$

13. San Fratello, N $37^{\circ} 55.849^{\prime}$ E $14^{\circ} 38.297^{\prime}, 1469 \mathrm{~m}$ 01. 05. 2013

14. San Fratello, N $37^{\circ}$ 57.299' E $14^{\circ} 37.327^{\prime}, 1019$ m 04. 05. 2013

15. San Fratello, N $37^{\circ} 56.559^{\prime}$ E $14^{\circ} 37.558^{\prime}, 1166 \mathrm{~m}$ 04. 05. 2013

16. San Fratello, N $37^{\circ} 56.559^{\prime}$ E $14^{\circ} 37.558^{\prime}, 1166$ m 05. 05. 2013

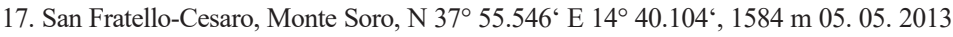

18. San Fratello, N $37^{\circ} 56.803^{\circ} \mathrm{E} 14^{\circ} 37.549^{`}, 1139 \mathrm{~m} \mathrm{05.05.} 2013$

19. San Fratello, N $37^{\circ} 56.803^{\circ} \mathrm{E} 14^{\circ} 37.547^{\circ}, 1143 \mathrm{~m} \mathrm{11.05.} 2013$

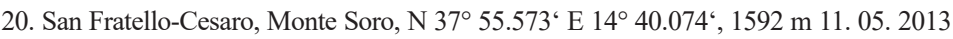

21. San Fratello, N $37^{\circ} 56.559^{`} \mathrm{E} 14^{\circ} 37.558^{`}, 1166 \mathrm{~m} 11.05 .2013$

22. Vall. San Giacomo, Zafferana Etnea, N $37^{\circ} 42.124^{`}$ E $15^{\circ} 5.701^{`}, 750 \mathrm{~m}$ 12. 05.2013

23. Fornazzo: Etna, 23. N $37^{\circ} 46.252^{\prime}$ E $15^{\circ} 4.947^{\prime}, 1459$ m 12. 05. 2013

24. Fornazzo: Etna, $\mathrm{N} 37^{\circ} 46.421^{\prime}$ E $15^{\circ} 3.978^{\prime}, 1600 \mathrm{~m} \mathrm{12.05.} 2013$

25. San Fratello, N $37^{\circ} 56.803^{\circ}$ E $14^{\circ} 37.547^{\circ}, 1143 \mathrm{~m} \mathrm{18.05.} 2013$

26. San Fratello, N $37^{\circ} 56.559^{`} \mathrm{E} 14^{\circ} 37.558^{\circ}, 1166 \mathrm{~m} \mathrm{18.05.} 2013$

27. Cefalu, Rocca di Cefalu, 19. 05. 2013 around $38^{\circ} 2^{\circ} 16.43^{\prime \prime} \mathrm{N}, 14^{\circ} 11^{\circ} 27.56^{\prime \prime} \mathrm{E}$

28. Capo di Milazzo, 09. 06. $201338^{\circ} 16^{\prime} 12.18^{\prime \prime} \mathrm{N}, 15^{\circ} 13^{\prime} 47.13^{\prime \prime} \mathrm{E}$

29. Capo di Milazzo, 11. 06. $201338^{\circ} 16^{\prime} 12.18^{\prime \prime} \mathrm{N}, 15^{\circ} 13^{\prime} 47.13^{\prime \prime} \mathrm{E}$

30. Capo di Milazzo, 14. 06. $201338^{\circ} 16^{\prime} 12.18^{\prime \prime} \mathrm{N}, 15^{\circ} 13^{\prime} 47.13^{\prime \prime} \mathrm{E}$

31. San Fratello, N $37^{\circ} 56.803^{\circ} \mathrm{E} 14^{\circ} 37.549^{`}, 1141 \mathrm{~m} \mathrm{15.06.} 2013$

32. San Fratello, N $37^{\circ} 56.559^{\circ}$ E $14^{\circ} 37.558^{`}, 1166 \mathrm{~m} \mathrm{15.06.} 2013$

33. San Fratello, N $37^{\circ} 57.301^{\prime}$ E $14^{\circ} 37.324^{`}, 1024$ m 15. 06. 2013

34. Capo di Milazzo , 17. 08. $201338^{\circ} 16^{\prime} 7.78^{\prime \prime} \mathrm{N}, 15^{\circ} 13^{\prime} 30.85^{\prime \prime} \mathrm{E}$

35. San Fratello, N $37^{\circ} 56.803^{\circ} \mathrm{E} 14^{\circ} 37.549^{`}, 1141 \mathrm{~m} 24.08 .2013$

\section{Results}

\section{List of species}

\section{Symphyta}

\section{Tenthredinidae}

Tenthredopsis scutellaris (Fabricius, 1804): San Fratello, 1143 m, 18. 05. 2013, 1 female, 3 males; San Fratello-Cesaro: Monte Soro 1592 m, 11. 05. 2013, 5 males. 


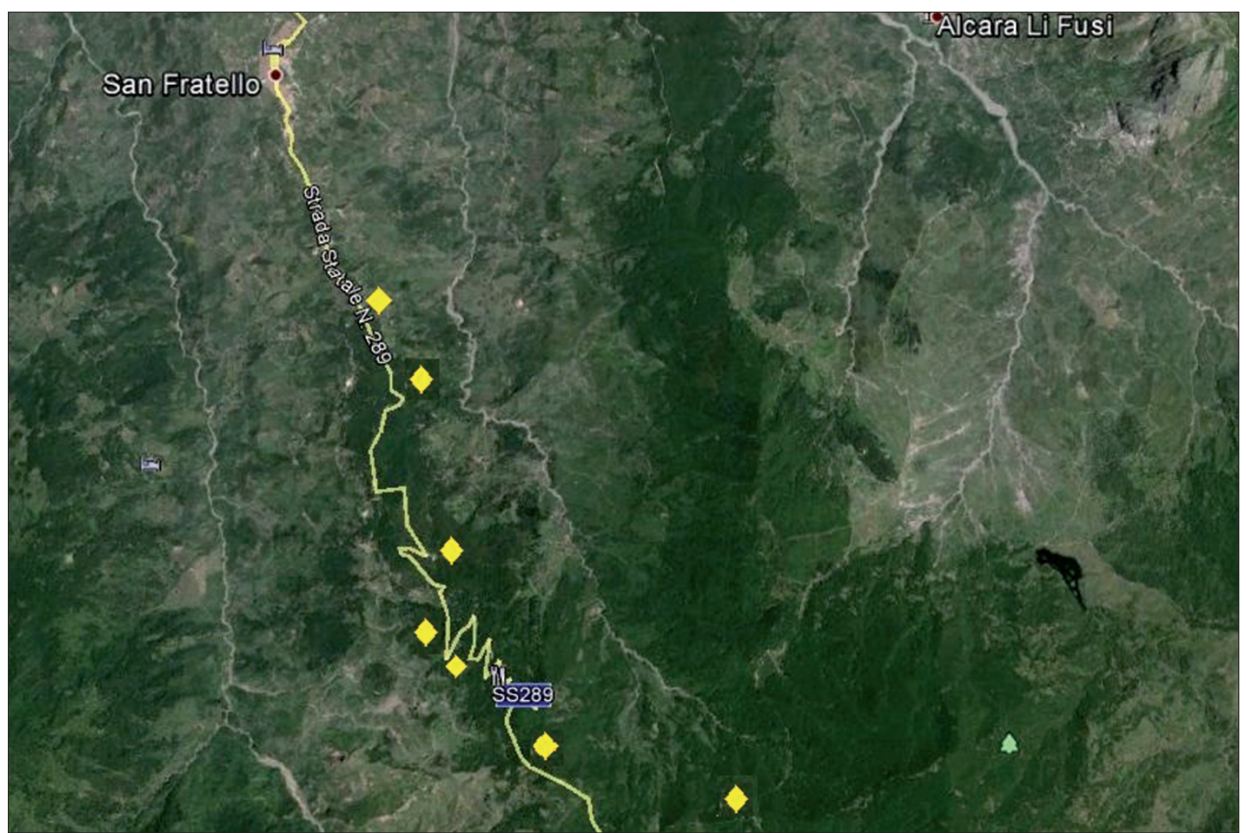

Fig. 1: Map of collecting sites between San Fratello and Cesaro in Monti Nebrodi

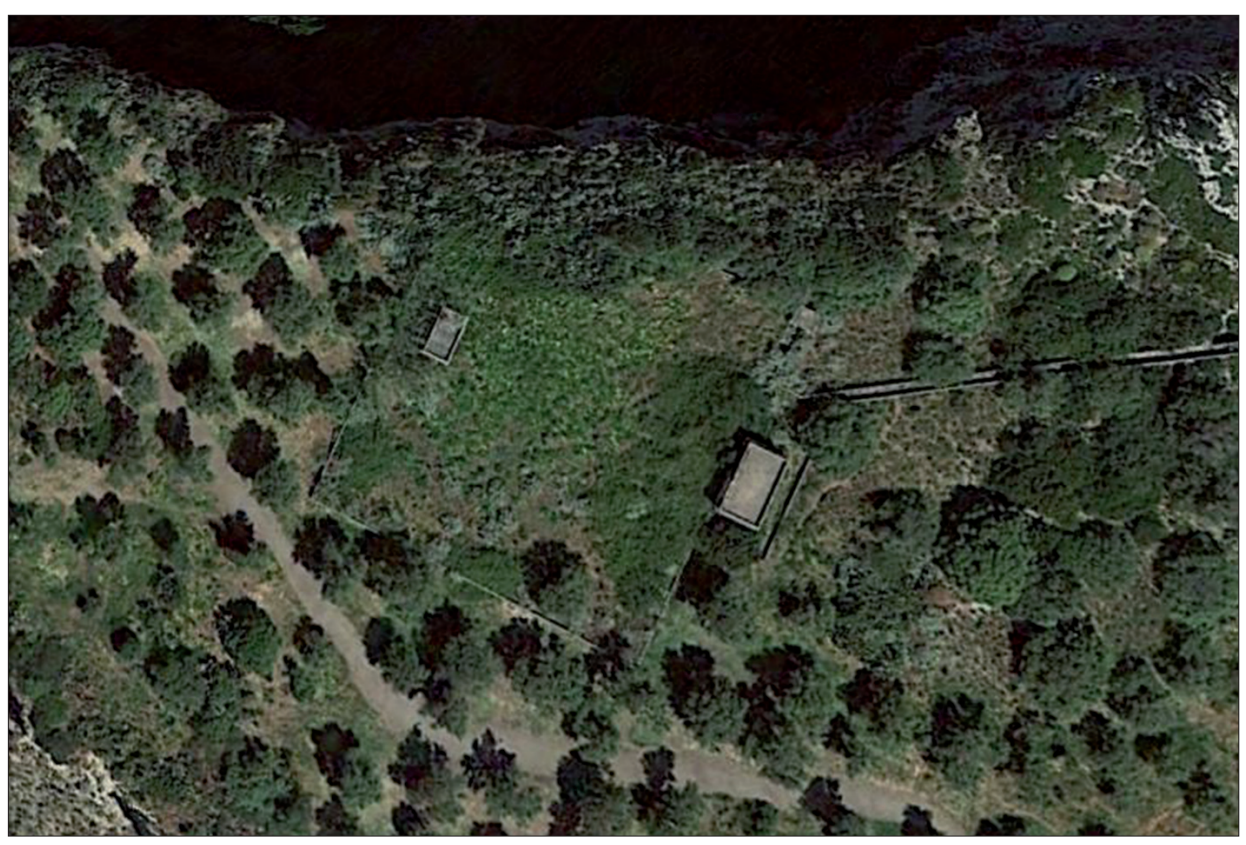

Fig. 2: Areal view of collection site at Capo di Milazzo close to lighting tower "Il Faro" 


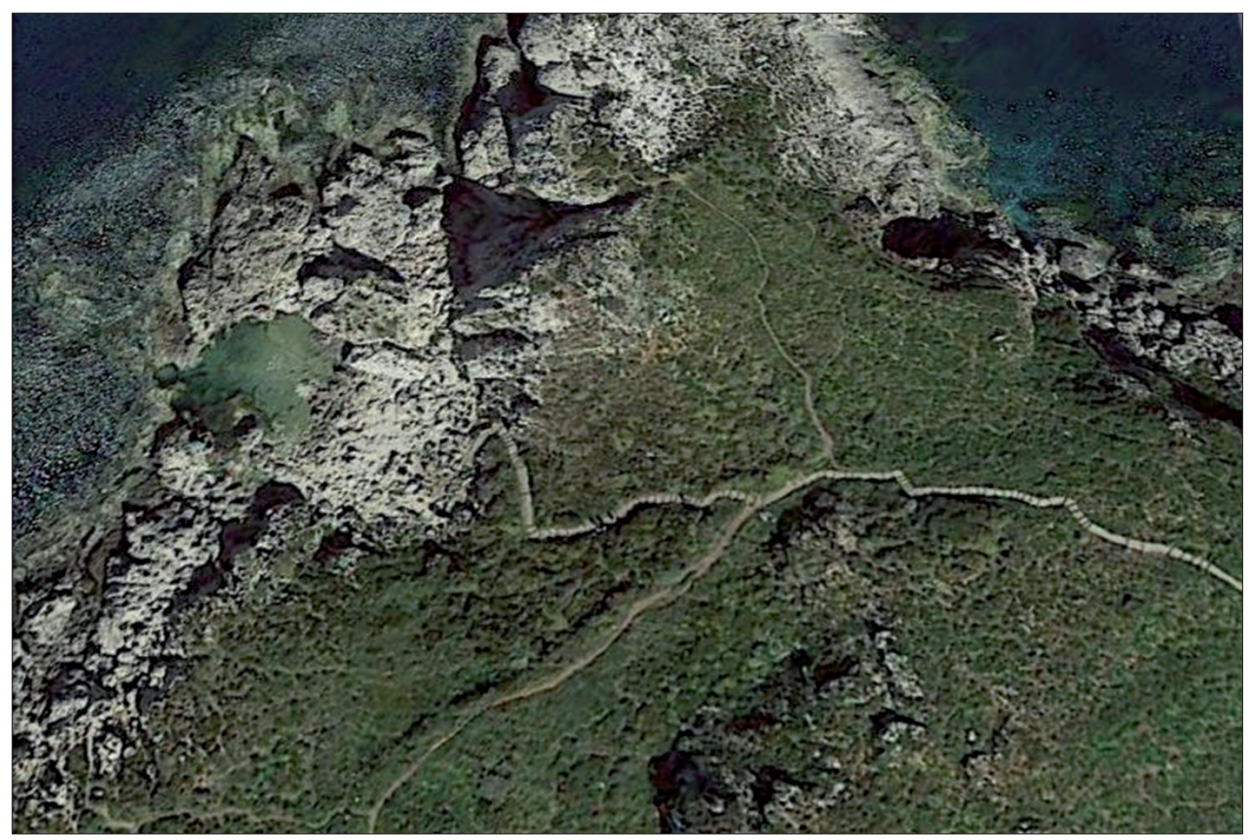

Fig. 3: Areal view of the second collecting site at Capo di Milazzo

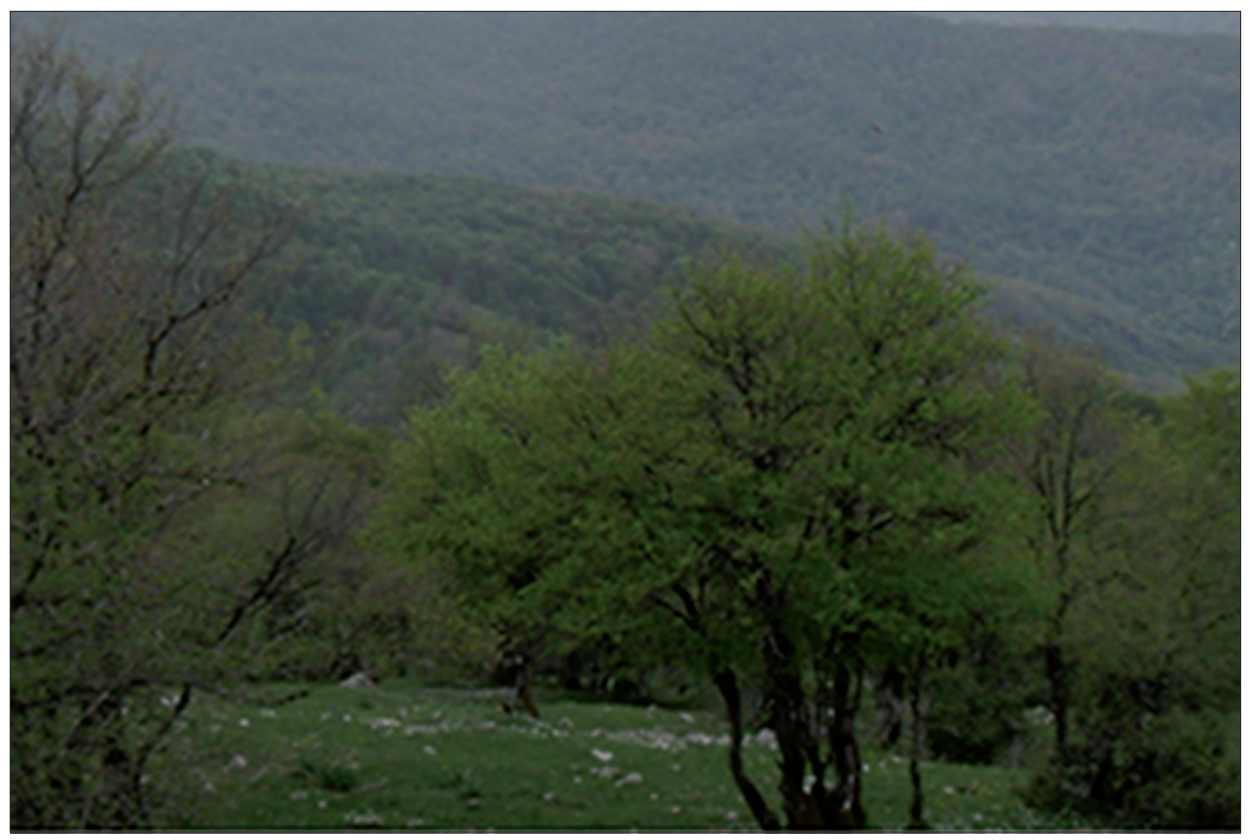

Fig. 4: The Monte Soro in Monti Nebrodi 


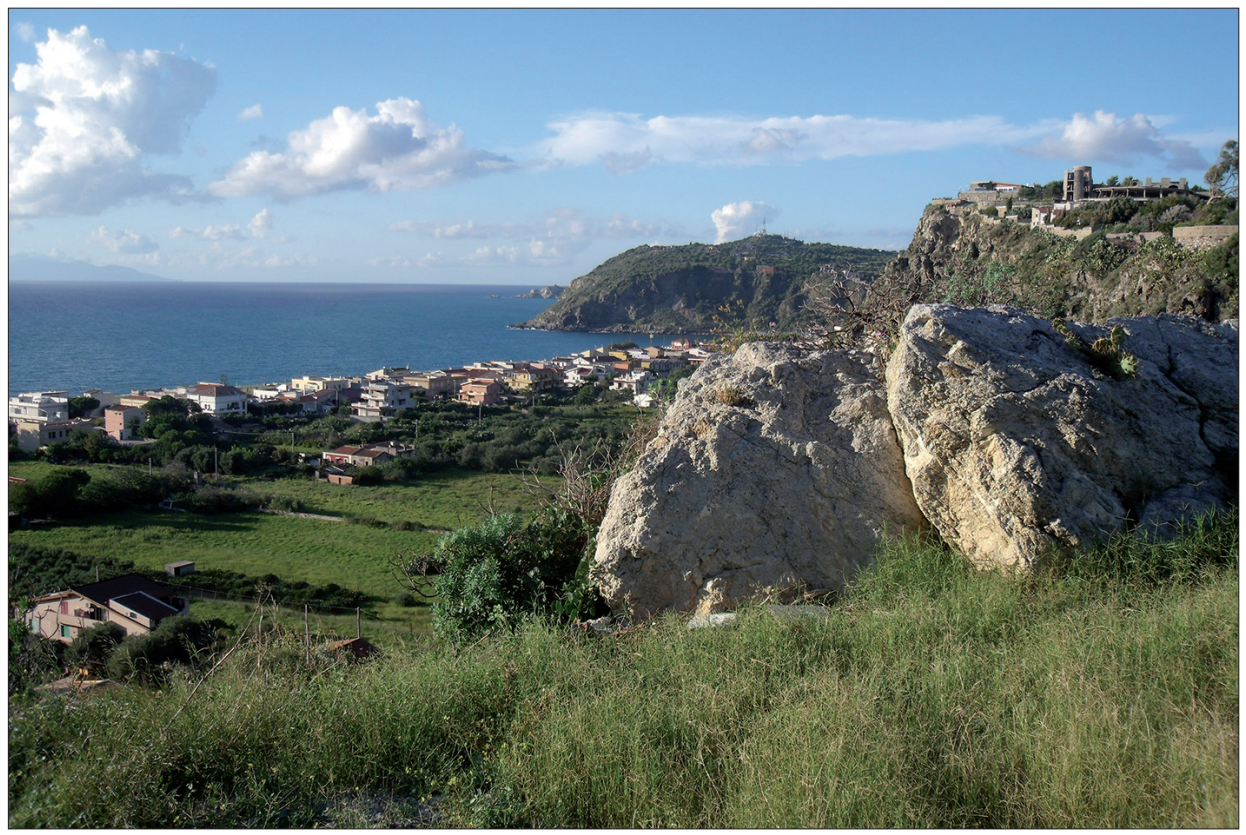

Fig. 5: Capo di Milazzo

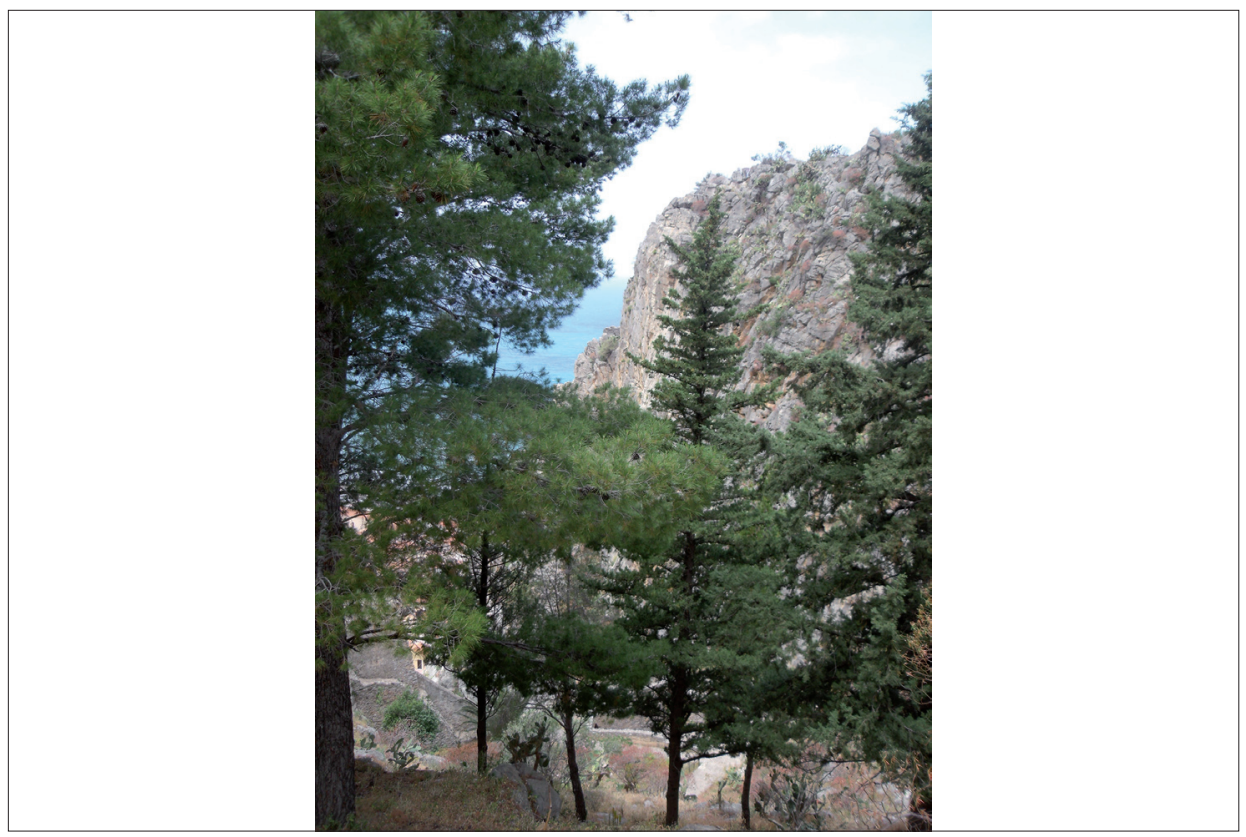

Fig. 6: Rocca di Cefalu 


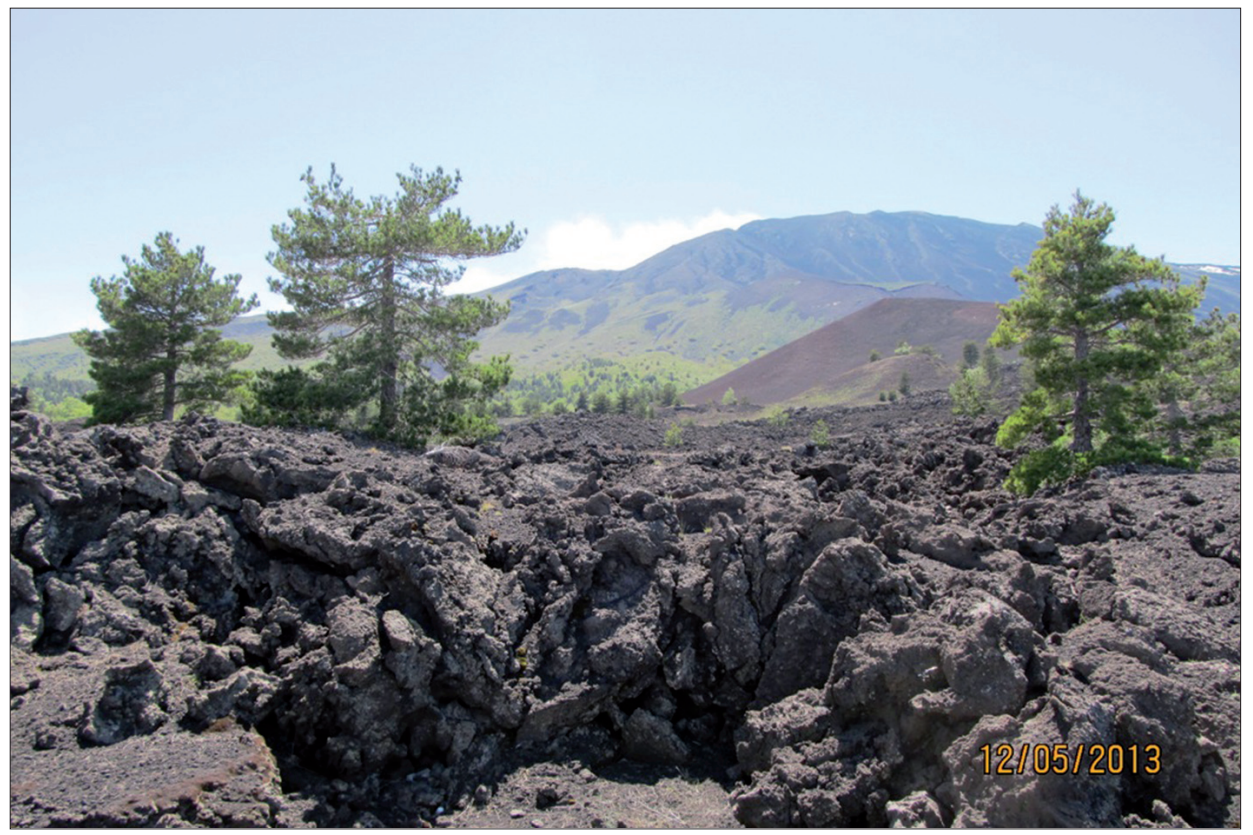

Fig. 7: On the Etna

Strongylogaster multifasciata (Geoffroy, 1785): San Fratello-Cesaro Monte Soro 1584 m, 05. 05. 2013, 1 female, 1 male; Patti, 472 m, 20. 04. 2013, 1 female; San Fratello 1143 m, 11. 05. 2013, 1 male, San Fratello 1374 m, 27. 04. 2013, 1 male; Fornazzo 1600 m, 12. 05. 2013, 1 male.

Aneugmenus padi (Linné 1761): San Fratello 1166 m, 05. 05. 2013, 2 males, 11. 05. 2013, 1 female, 1 male, 18. 05. 2013, 2 females, 1 male; San Fratello 1010 m, 01. 05. 20131 male; San Fratello1469 m, 01. 05. 2013, 1 female; Patti, 472 m, 20. 04. 2013, 1 female, 3 males; San Fratello-Cesaro Monte Soro 1584 m, 05. 05. 2013, 1 male.

Empria liturata (Gmelin, 1790): San Fratello 1166 m, 11. 05. 2013, 1 female, San Fratello 1469 m, 01. 05. 2 males.

Athalia circularis (Klug, 1815): San Giacomo, Zafferana Etnea 750 m, 12. 05. 2013, 1 male.

Athalia cordata Serville, 1823: San Fratello 1380 m, 27. 04. 2013, 2 males; San Fratello 1143 m, 11. 05. 2013, 1 male.

Pristiphora (Pristiphora) armata (Thomson, 1863): San Fratello-Cesaro Monte Soro 1584 m, 05. 05. 2013, 1 female.

Stromboceros delicatulus (Fallén, 1808): San Fratello 1166 m, 05. 05. 2013, 1 female.

Macrophya (Macrophya) annulata (Geoffroy, 1785): San Fratello 1143 m, 18. 05. 2013, 1 female.

Macrophya (Macrophya) montana (Scopoli, 1763): Vall. San Giacomo, Zafferana Etnea 750 m, 12. 05. 2013, 1 female, 1 male.

\section{Cephidae}

Calameuta (Calameuta) pallipes (Klug, 1803): Vall. San Giacomo, Zafferana Etnea $750 \mathrm{~m}, 12.05 .2013,2$ males. 


\section{Aculeata}

\section{Chrysididae}

Pseudomalus auratus (Linnaeus, 1758): Capo di Milazzo 28 m, 14. 06. 2013, 2 females.

\section{Mutillidae}

Physetopoda lucasii (Smith, 1855): Capo di Milazzo 28 m, 09. 06. 2013, 1 male. Mutilla quinquemaculata Cyrillo, 1787: Capo di Milazzo 128 m, 01. 06. 2013, 1 male.

\section{Scoliidae}

Scolia carbonaria Linnaeus, 1767: Capo di Milazzo 28 m, 09. 06. 2013, 1 male, 14. 06. 2013, 1 male, 17. 08. 2013, 1 male.

Megascolia flavifrons ssp. flavifrons (Fabricius, 1775): Milazzo: Raffineria di Milazzo, 14. 05. 2013, 1 female.

\section{Pompilidae}

Anospilus orbitalis Costa, 1863: Capo di Milazzo 28 m, 11. 06. 2013, 1 female. Aporus fulviventris spp. pollux (Kohl, 1888): Capo di Milazzo 28 m, 14. 06. 2013, 1 female. Auplopus rectus (Haupt, 1926): Capo di Milazzo 28 m, 09. 06. 2013, 1 female. Dicyrtomellus tingitanus (Wolf, 1966): Capo di Milazzo 28 m, 09. 06. 2013, 1 male. Anospilus orbitalis (Costa, 1863): Capo di Milazzo 28 m, 11. 06. 2013, 1 female. Priocnemis coriacea Dahlbom, 1843: San Fratello 1139 m, 05. 05. 2013, 1 male. Priocnemis mimula Wesmael, 1851: San Fratello 1139 m, 05. 05. 2013, 1 female, 1168 m 01. 05. 2013, 1 female; San Fratello-Cesaro Monte Soro 1592 m, 11. 05. 2013, 1 female.

\section{Vespidae}

Polistes dominulus (Christ, 1791): Capo di Milazzo 28 m, 09. 06. 2013, 1 female. Eumenes mediterraneus Kriechbaumer, 1879: Capo di Milazzo 28 m, 09. 06. 2013, 1 female, 11. 06. 2013, 1 female, 1 male.

Eumenes lunulatus Fabricius, 1804: Capo di Milazzo 28 m, 11. 06. 2013, 1 male.

\section{Sphecidae}

Ammophila heydeni Dahlbom, 1845: Capo di Milazzo 28 m, 11. 06. 2013, 1 male.

\section{Crabronidae}

Crossocerus cetratus (Shuckard, 1837): San Fratello-Cesaro Monte Soro 1584 m, 05. 05. 2013, 1 female.

Crossocerus pusillus Lepeletier and Brullé, 1834: Fornazzo 1459 m, 12. 05. 2013, 1 male. Oxybelus mucronatus (Fabricius, 1793): Capo di Milazzo 28 m, 09. 06. 2013, 1 male.

Philanthus triangulum (Fabricius, 1775): Capo di Milazzo 28 m, 09. 06. 2013, 2 females, 11. 06. 2013, 1 female.

Trypoxylon figulus (Linnaeus, 1758): San Fratello-Cesaro Monte Soro 1592 m, 11. 05. 2013, 1 male.

\section{Andrenidae}

Andrena agilissima (Scopoli, 1770): Fornazzo 1600 m, 12. 05. 2013, 1 male. Andrena alutacea Stöckhert, 1942: San Fratello 1166 m, 15. 06. 20131 female. Andrena chrysosceles (Kirby, 1802): San Fratello 1141 m, 15. 06. 2013, 1 male. Andrena combinata (Christ, 1791): Capo di Milazzo 28 m, 09 06. 2013, 1 female. Andrena flavipes Panzer, 1799: San Fratello 1139 m, 05. 05. 2013, 1 male, 1592 m ,11. 05. 2013, 1 male; San Fratello-Cesaro Monte Soro 1584 m, 05. 05. 2013, 1 male, 11. 05. 2013, 1 male. 
Andrena floricola Eversmann, 1852: San Cosimo 587 m, 20. 04. 1013. 1 female. Andrena fulva (Müller, 1766): San Fratello 1380 m, 27. 04. 2013, 1 female.

Andrena fulvata Stöckhert, 1930: San Fratello 871 m, 01. 05. 2013, 1 female; San

Fratello-Cesaro Monte Soro 1592 m, 11. 05. 2013, 1 female.

Andrena fuscosa Erichson, 1835: Capo di Milazzo 28 m, 17. 08. 2013, 2 males.

Andrena gelriae van der Vecht, 1927: San Fratello 01. 05. 2013, 1 male.

Andrena hesperia Smith, 1853: San Fratello 1166 m, 05. 05. 2013, 1 female.

Andrena humilis Imhoff, 1832: San Cosimo 587 m, 20. 04. 2013, 1 male.

San Fratello 1141 m, 15. 06. 2013, 3 females, 18. 05. 2013, 1 female, 1166 m, 05. 05. 2013, 1 female, 1139 m, 05. 05. 2013, 7 males, 1019 m, 04. 05. 2013, 1 male; San Fratello-Cesaro Monte Soro 1584 m, 05. 05. 2013, 1 female, 1 male, 1592 m, 11. 05. 2013, 5 males.

Andrena labialis (Kirby, 1802): San Fratello 1166 m, 15. 06. 2013, 1 female, 1 male.

Andrena labiata Fabricius, 1781: San Fratello 1166 m, 18. 05. 2013, 1 male, San Fratello-Cesaro Monte Soro 1592 m, 11. 05. 2013, 1 male.

Andrena lagopus Latreille, 1809: Blaida 579 m, 20. 04. 2013, 1 female.

Andrena minutula (Kirby, 1802): San Fratello 1141 m, 15. 06. 2013, 1 female.

Andrena similis Smith, 1849: Capo di Milazzo 28 m, 09. 06. 2013, 1 female 1 male.

Andrena simontornyella Noskiewicz, 1939: San Fratello 808 m, 27. 04. 2013, 2 females, 1037 m, 27. 04. 2013, 1 female; San Fratello-Cesaro Monte Soro 1584 m, 05. 05. 2013, 1 female.

Andrena thoracica (Fabricius, 1775): San Fratello 1166 m, 15. 06. 2013, 1 female.

Panurgus cavannae Gribodo, 1880: Cefalu, Rocca di Cefalu 23 m, 19. 05. 2013, 1 male, San Fratello 1024 m, 15. 06. 1 female, 2 males, 1141 m 15. 06. 2013, 1 female; Vall. San Giacomo Zafferana Etnea 750 m, 12. 05. 2013, 2 males.

\section{Apidae}

Anthophora retusa (Linné, 1758): San Fratello-Cesaro Monte Soro 1592 m, 11. 05. 2013, 1 female.

Apis mellifera Linnaeus, 1758: San Fratello 1037 m, 20. 04. 2013, 1 female, 2 males.

Bombus muscorum (Linnaeus, 1758): San Fratello 1141 m, 24. 08. 2013, 1 female.

Bombus pratorum (Linnaeus, 1761): San Fratello 871 m, 01. 05. 2013, 1 female; 1166 m 18. 05. 2013, 2 males.

Bombus terrestris (Linnaeus, 1758): San Fratello 1141 m, 15. 06. 2013, 1 male.

Psithyrus rupestris (Fabricius, 1793): San Fratello 1024 m, 15. 06. 2013, 1 male.

Ceratina cucurbitina (Rossi, 1792): Acquedolci 27. 04. 2013, 1 male; Blaida 579 m, 20. 04. 2013, 3 females, 2 males; San Fratello 808 m, 27. 04. 2013, 2 males, 1019, m, 04. 05. 2013, 1 male, $1037 \mathrm{~m}, 27.04 .1$ male, $1139 \mathrm{~m}, 05.05 .1$ male, $1166 \mathrm{~m}, 04.05 .2013,1$ male, 1439 m 1 male; San Fratello-Cesaro Monte Soro 1592 m, 11. 05. 2013, 3 males.

Eucera eucnemidea Dours, 1873: San Fratello 1024 m, 15. 06. 2013, 1 female, 1 male, $1141 \mathrm{~m}, 15.06 .2013,6$ females, $1143 \mathrm{~m}, 18.05 .2013,1$ male, $1166 \mathrm{~m}, 05.05 .2013,3$ males, 1374 m, 27. 04. 2013, 1 male; San Fratello-Cesaro Monte Soro 1584 m, 05. 05. 2013, 2 males, $1592 \mathrm{~m}, 11.05 .2013,1$ male.

Eucera nigrescens Pérez, 1879: Blaida 579 m, 20. 04. 2013, 1 male; San Cosimo 587 m, 20. 04. 2013, 1 female 2 males.

Eucera parvula Friese, 1895: San Fratello 1024 m, 15. 06. 2013, 1 female; San Fratello-Cesaro Monte Soro 1584 m, 05. 05. 2013, 1 female.

Melecta luctuosa (Scopoli, 1770): San Fratello, 27. 04. 2013, 1 female, 1469 m, 01. 05. 2013, 1 female. 
Nomada conjungens Herrich-Schaeffer, 1839: San Fratello-Cesaro Monte Soro 1592 m, 11. 05. 2013, 2 males.

Nomada fabriciana (Linnaeus, 1767): San Fratello 1143 m, 11. 05. 2013, 1 female, 1469 m, 01. 05. 2013, 1 male.

Nomada femoralis Morawitz, 1869: San Fratello 1166 m, 04. 05. 2013, 1 male; San Fratello-Cesaro Monte Soro 1592 m, 11. 05. 2013, 2 males.

Nomada flavoguttata (Kirby, 1802): San Fratello 1139 m, 05. 05. 2013, 1 male; San Fratello-Cesaro Monte Soro 1592 m, 11. 05. 2013, 1 male.

Nomada fucata Panzer, 1798: San Fratello 1139 m, 05. 05. 2013, 1 male.

Nomada glaucopis Pérez, 1884: San Fratello-Cesaro Monte Soro 1584 m, 05. 05. 2013, 1 female.

Nomada sheppardana (Kirby, 1802): San Fratello 1166 m, 04. 04. 2013, 1 female; San Fratello-Cesaro Monte Soro 1584 m, 05. 05. 2013, 1 male.

Nomada thersites Schmiedeknecht, 1882: San Fratello-Cesaro Monte Soro1592 m, 11. 05. 2013, 1 male.

\section{Colletidae}

Colletes nigricans Gistel, 1857: Capo di Milazzo 28 m, 14. 06. 2013, 1 male.

Hylaeus clypearis (Schnck, 1853): Capo di Milazzo 28 m, 09. 06. 2013, 1 female, 1 male, 14. 06. 2013, 1 male.

Hylaeus communis Nylander, 1852: San Fratello 1166 m, 18. 05. 2013, 1 female.

Hylaeus gibbus Saunders, 1856: San Fratello $1141 \mathrm{~m}, 15.06 .2013,1$ male.

Hylaeus gredleri Förster, 1871: Capo di Milazzo 28 m, 09. 06. 2013, 1 male; San Fratello $1141 \mathrm{~m}, 15.06 .2013,1$ male.

Hylaeus imparilis Förster, 1871: Capo di Milazzo 28 m, 09. 06. 2013, 1 female, 17. 08. 2013, 1 female.

Hylaeus punctatus (Brullé, 1832): Capo di Milazzo 28 m, 11. 06. 2013, 1 female, 2 males, 09. 06. 2013, 1 female, 1 male.

\section{Halictidae}

Halictus asperulus Pérez, 1895: Capo di Milazzo 28 m, 09. 06. 2013, 1 female, 11. 06. 2013, 1 male.

Halictus fulvipes (Klug, 1817): Capo di Milazzo 28 m, 17. 08. 2013, 2 females, Cefalu, Rocca di Cefalu 23 m, 19. 05. 2013, 2 females.

Halictus pollinosus Sichel, 1860: Capo di Milazzo 28 m, 14. 06. 2013, 1 male.

Halictus quadricinctus (Fabricius, 1776): Capo di Milazzo 28 m, 17. 08. 2013, 1 female, 3 males.

Halictus scabiosae (Rossi, 1790): Capo di Milazzo 28 m, 11. 06. 2013, 2 males, 17. 08. 2013, 1 female.

Halictus sp. (eurygnathus group): Capo di Milazzo 28 m, 17. 08. 2013, 1 male.

Lasioglossum albipes (Fabricius, 1781): San Fratello 1166 m, 05. 05. 2013, 1 female; Vall. San Giacomo, Zafferana Etnea 750 m, 12. 05. 2013, 1 male.

Lasioglossum albocinctum (Lucas, 1846): San Fratello 1024 m, 15. 06. 2013, 1 female. Lasioglossum crassepunctatum (Blüthgen, 1923): San Fratello 808 m, 27. 04. 2013, 1 female. Lasioglossum cristula (Pérez, 1895): San Fratello 871 m, 01. 05. 2013, 1 female.

Lasioglossum interruptum ssp. opacum (Pérez, 1895): Capo di Milazzo 28 m, 17. 08. 2013, 2 females; San Cosimo 587 m, 20. 04. 2013, 1 female.

Lasioglossum laeve (Kirby, 1802): San Fratello 1024 m, 15. 06. 2013, 1 female.

Lasioglossum laevigatum (Kirby, 1802): San Fratello 1166 m, 15. 06 2013, 1 female. Lasioglossum laticeps (Schenck, 1969): San Fratello 1019 m, 04. 05. 2013, 1 female. 
Lasioglossum leucozonium (Schrank, 1781): San Fratello 1141 m, 15. 06. 2013, 1 female. Lasioglossum nitidiusculum (Kirby, 1802): Blaida 579 m, 20. 04. 2013, 1 female. Lasioglossum punctatissimum (Schenck, 1853): San Fratello 1469 m, 01. 05. 2013, 1 female. Lasioglossum puncticolle (Morawitz, 1872): San Fratello 1141 m, 24. 08. 2013, 2 females. Lasioglossum trichopygum (Blüthgen, 1923): Capo di Milazzo 28 m, 11. 06. 2013, 1 male. Lasioglossum xanthopus (Kirby, 1802): San Fratello 1166 m, 05. 05. 2013, 1 female. Lasioglossum villosulum (Kirby, 1802): San Fratello 1141 m, 15. 06. 2013, 1 female. Lasioglossum (Evylaeus) sp.: San Fratello 808 m, 17. 27. 04. 2013, 1 female, 1141 m, 15. 06. 2013, 1 female.

Pseudapis unidentata (Olivier, 1811): Capo di Milazzo 28 m, 17. 08. 2013, 1 female. Sphecodes gibbus (Linnaeus, 1758): Capo di Milazzo 28 m, 09. 06. 2013, 1 male. Sphecodes rufivetris (Panzer, 1798): Capo di Milazzo 28 m, 09. 06. 2013, 1 male. Sphecodes sp.: San Fratello 1166 m, 11. 05. 2013, 1 male.

\section{Megachilidae}

Anthidium manicatum (Linnaeus, 1758): Capo di Milazzo 28 m, 17. 08. 2013, 1 female.

Anthidium septemdentatum Latreille, 1809: Cefalu, Rocca di Cefalu 23 m, 19. 05. 2013, 1 female.

Osmia niveata (Fabricius, 1804): San Fratello 1143 m, 18. 05. 2013, 1 female; Vall. San Giacomo Zafferana Etnea 750 m, 12. 05. 2013, 1 male.

Osmia bicornis (Linnaeus, 1758): San Fratello 1469 m, 01. 05. 2013, 1 female.

Chelostoma florisomne (Linnaeus, 1758): San Fratello-Cesaro Monte Soro $1592 \mathrm{~m}$, 11. 05. 2013, 1 male; San Fratello 1166 m, 04. 05. 2013, 1 male.

Chelostoma stefanii Nobile, 1995: San Fratello 1469 m, 01. 05. 2013, 1 female, 04. 05. $1466 \mathrm{~m}, 1$ female.

Chelostoma emarginatum (Nylander, 1856): San Fratello 808 m, 27. 04. 2013, 1 female; San Fratello 1139 m, 05. 05. 2013, 1 male, 1166 m, 05. 05. 2013, 2 males, 18. 05. 2013, 1 male; San Fratello 1595 m, 11. 05. 2013, 1 male.

\section{Results}

111 species were recorded in the following classification: 11 Symphyta: 10 Tenthredinidae and 1 Cephidae species and 100 Aculeata species: 79 wild bees (Apoidea s. str.), 1 Sphecidae, 5 Crabronidae, 3 Vespidae, 1 Chrysididae, 2 Scoliidae, 2 Mutillidae and 7 Pompilidae species.

\section{Some interesting and new species}

Empria liturata (Gmelin, 1790): New record for Sicily. Far the commonest Empria species in Europe. Subrecently reported from Vietnam either. Ocasional insect pest of strawberry in horticulture. Known hostplants: Fragaria and Geum spp.

Andrena alutacea Stöckhert, 1942: Rare species, native in Austria; Bulgaria; Croatia; Czech Republic; France; Germany; Greece (East Aegean Is., Greece (mainland)); Italy (including Sicily); Latvia; Liechtenstein; Macedonia, the former Yugoslav Republic; Poland; Romania; Slovenia; Spain and Switzerland (IUCN Red List, 2017). Subrecently revised and separated from Andrena proxima (Kirby, 1802) (SCHMID-EGGER 2005).

Chelostoma stefanii Nobile, 1995: Subrecently described species. Probably endemic in Sicily. It seems not to be rare. This species described from the Nebrodi Mountains, 
from C. da Pomarazzo, at 1550m. Chelostoma siciliae Müller, 2012 is synonim of this species and described from the Madonnie Mountains from Collesno below $600 \mathrm{~m}$.

Nomada glaucopis Pérez, 1884: Rare species, new record for Sicily. Known from

France, the Italian mainland, Sardinia and North Africa (ACHTERBERG 2013).

Nomada thersites Schmiedeknecht, 1882: Known from Cyprus, Southern part of European Russia, Slovakia, Sicily and the Near East (ACHTEBERG 2013). Rare species.

\section{Dominant species}

In the Nebrodi Mountains, the following species were the most frequent: Aneugmenus padi (LinNé 1761) with 14, Andrena humilis Imhoff, 1832 with 21, Ceratina cucurbitina (Rossi, 1792) with 15 and Eucera eucnemidea Dours, 1873 with 17 collected specimens.

In the Milazzo Peninsula we did not collected from any species more than 4 specimens. Most of the species were represented only by 1 single or 2 specimens.

\section{Acknowledgement}

Authors express their grateful thanks for their support in identification of some complicated Aculeata taxon to Dr. Fabrizio Turrisi, to Dr. Vittorio Nobile and to Dr. Andreas Müller.

\section{References}

ACHTERBERG, C. 2013: Hymenoptera in Fauna Europaea version 2.6.2. http://www.faunaeur.org . last accessed $10^{\text {th }}$ August 2017.

IUCN Global Species Programme Red List Unit, 2017: The IUCN Red List of Threatened Species. Version 2017-1. <www.iucnredlist.org>. Downloaded on 10 August 2017.

Liston, A., JaCobs, H. J. \& Turrisi, G. F. 2013: New data on the sawfly fauna of Sicily (Hymenoptera, Symphyta: Xyeloidea, Tenthredinoidea, Pamphilioidea, Cephoidea, Orussoidea). - Entomologists's Monthly Magazine 149: 29-65.

NobiLe V., 1988: Contributo alla conoscenza degli Apoidei (Insecta, Hymenoptera) di Sicilia. II. Il genere Osmia Panzer, 1806. - Animalia 15: 159-173.

Nobile, V. 1989: Il genere Anthidium Fabricius, 1804 e affini (Hymenoptera, Apoidea, Megachilidae) in Sicilia, con descrizione di Pseudanthidium alpinum gregoriense subsp. n. - Animalia 16: 131-145.

NobILE, V., 1990: Contributo alla conoscenza delle Api parassite (Insecta, Hymenoptera) di Sicilia. II. Il genere Nomada Scopoli, 1770 con descrizione una nuove specie. - Animalia 17: 219-243

Nobile, V., 1995a: Api (Insecta, Hymenoptera) nuove o poco note di Sicilia e Sardegna. - Bollettino dell'Accademia Gioenia di Scienze naturali in Catania, 28: 147-159.

NobiLe, V., 1995b: The apidological situation in Sicily, with remarks on the phenomenon of endemism, pp. 127-146. - In: BanaszaK, J. (ed.), Changes in fauna of wild bees in Europe. Pedagogical University, Bydgoszcz.

Nobile, V. \& Campadelli, G. 1998: II genere Sphecodes Latreille, 1804 in Italia (Hymenoptera, Apoidea, Halictidae). - Bollettino dell'Istituto di Entomologia Guido Grandi della Università degliStudi di Bologna 52: 85-103.

Nobile, V., Meloni, C. \& Tomarchio, S. 2005: New Andrena species from Sicily and Sardinia Hymenoptera, Andrenidae I Andrena nuove per la Sicilia e la Sardegna Hymenoptera, Andrenidae I. - Bollettino della Societa Entomologica Italiana. settembre-dicembre 1373: 223-228.

Nobile, V. \& Tomarchio, S. 1998: Contributo alla conoscenza degli Apoidei di Sicilia. XV. Il genere Spatulariella Popov, 1939 (Hymenoptera Colletidae, Hylaeinae) con descrizione di una nuova specie. Bollettino Accademia Gioenia di Scienze naturali in Catania 30: 291-298. 
NobiLe, V. \& Turrisi G. F., 1997: Contributo alla conoscenza degli Apoidei di Sicilia. XIII. Le tribù Lithurgini, Dioxyni, Stelidini (Insecta, Hymenoptera, Megachilidae). - Bollettino dell'Accademia Gioenia Scienze Naturali, Catania 29(351) (1996): 15-26.

Nobile, V. \& Turrisi, G. F., 1999: Contributo alla conoscenza delle Api parassite di Sicilia. La tribù Coelyoxini (Hymenoptera, Apoidea, Megachilidae). - Bollettino dell'Accademia Gioenia di Scienze Naturali, Catania 32(356): 161-170.

Nobile, V. \& Turrisi, G. F., 2004: Contribution to the knowledge of Italian cleptoparasitic bees. X. The genus Sphecodes Latreille, "pinguicolus Pérez" group, with desription of new species (Hymenoptera, Apoidea, Halictidae). - Entomofauna 25 (8): 117-132.

Nobile, V. \& Turrisi, G. F., 2015: New or little known Halictidae from Italy (Hymenoptera, Apoidea). Bolletino della Società Entomologica Italiana 147 (1): 39-42

Pagliano, G., 1985: Sphecinae italiani (Hymenoptera: Sphecidae) parte II. - Bollettino del Museo Regionale di Scienze Naturali Torino 3(1): 5-28.

Pagliano, G., 1986: Methochidae e Scolidae italiani (Hymenoptera). - Bollettino del Museo civico di Storia naturale di Venezia 37: 157-181.

Pagliano, G., 1988a: Catalogo degli Imenotteri italiani. I. Halictidae. - Bollettino del Museo civico di Storia naturale di Venezia 38: 85-128.

Pagliano, G., 1988b: Prospetto sistematico degli Apoidea italiani. - Annali Facoltà Scienze Agrarie , Università Torino 15: 97-128.

Pagliano, G., 1988c: Philantinae italiani (Hymenoptera: Sphecidae) parte II. - Bollettino del Museo Regionale di Scienze Naturali Torino 6(1): 133-174.

Pagliano, G., 1990: Catalogo degli Imenotteri Italiani. - Sphecidae. - Bollettino del Museo Regionale di Scienze Naturali Torino 8(1): 53-141.

Pagliano, G., 1992: Catalogo degli Imenotteri Italiani. III. Melittidae. - Bollettino della Società Entomologica Italiana Genova, 124(2): 133-138.

Pagliano, G., 1993: Catalogo degli Imenotteri Italiani. IV. (Apoidea: Colletidae, Andrenidae, Megachilidae, Anthophoridae, Apidae). - Memorie della Società Entomologica Italiana 72: 331-467.

Pagliano, G., 1994: Hymenoptera: Apoidea. In: Minelli A., Ruffo S., La Posta F., (Editori). Check-list delle specie della fauna italiana 106. - Officine Grafiche Calderini, Bologna. 25 pp.

Pagliano, G. \& Nobile V., 1993: Il genere Xylocopa Latreille 1802 in Italia (Hymenoptera: Apoidea). Bollettino dell'Accademia Gioenia di Scienze Naturali in Catania 26(342): 133-144.

Pagliano, G. \& Nobile, V. 1995: Il genere Nomioides Schenck 1866 in Italia (Hymenoptera, Apoidea, Halictidae). - Bollettino dell'Accademia Gioenia di Scienze Naturali in Catania 28(349): 547-561.

Pagliano, G. \& Strumia, F. 2007: Contributo alla conoscenza dei Mutillidae italiani (Hymenoptera, Scolioidea). - Bollettino del Museo regionale di Scienze naturali, Torino 24: 25-110.

Pesarini, F. \& Turrisi, G. F., 2001: Contributo alla conoscenza dei Sinfiti di Sicilia (Hymenoptera Symphyta). - Memorie della Società Entomologica Italiana 80: 183-221.

SCHMID-EgGer 2005: Proxiandrena subgen. nov. und Revision der West und Zentralpaläarktischen Arten der Andrena proxima-Gruppe (Hymenoptera, Apidae). - Revue Suisse de Zoologie 112: 1029-1044.

SoIKA, G. A. 1944: Risultati di raccolte imenotterologiche in Sicilia. - Istituto di Zoologia e di Anatomia Comparata dell'Università di Modena: 5-21.

SolKA, A. \& Borsato, W. 1995: Checklist delle specie della fauna Italiana, Hymenoptera Vespoidea 103. Herausgeber Ministero del Ambiente e Comitato Scientifico per la Fauna d'Italia.

Strumia, F., 2005: Hymenoptera Chrysididae. In: Ruffo S., \& Stoch F. (eds.), Checklist e distribuzione della fauna italiana. 10.000 specie terrestri e della acque interne. - Memorie del Museo Civico di Storia Naturale di Verona, serie 2, sezione Scienze della Vita 16: 269-270 + CD-ROM.

Tomarchio, S. \& Turrisi, G. F. 2006: New or little known Sphecidae (Hymenoptera Aculeata) from Sicily (Italy). - Linzer Biologische Beiträge 38 (1): 953-960.

TurRISI, G. F., 1999a: Contributo alla conoscenza dei Mutillidae di Sicilia ed isole circumsiciliane (Hymenoptera Scolioidea). - Bollettino dell'Accademia Gioenia di Scienze Naturali, Catania 31(354): 119-155.

TurrisI, G. F., 1999b: La famiglia Sapygidae in Sicilia (Hymenoptera Scolioidea). - Bollettino dell'Accademia Gioenia di Scienze Naturali, Catania 31(354) (1998): 335-338.

TurrisI, G. F. 1999c: Xyelidae, Aulacidae, Heloridae e Masaridae, quattro famiglie nuove per la fauna siciliana (Insecta Hymenoptera). - Bollettino della Società entomologica italiana 131(1): 41-46.

Turrisi, G. F., 2009: Faunsitic remarks on Sicilian Cleptes Latreille, with two new records (Hymenoptera Chrysididae Cleptinae) - Il Naturalista siciliano S. IV, 33(3-4): 295-304. 
TURRISI, G. F.: 2011: Diversity and biogeographical remarks on "Symphyta" of Sicily (Hymenoptera). Biogeographia 30: 511-528.

Turrisi, G. F. \& Olmi, M. 2009: New records of Drynidae from Sicily (Hymenoptera Chrysidoidea) - Il Naturalista Siciliano IV, 33(3-4), pp. 289-293.

Vicidomini, S., 2007: Distribuzione degli Xylocopini (Hymenoptera: Apidae: Xylocopinae) in Italia: il Parco del Vulcano Etna e aree limitrofe (provincia di Catania: Sicilia). - Il Naturalista Campano 44: 1-4.

Vicidomini, S., Pignataro, C. \& Insacco, G. 2005: Xylocopini (Hymenoptera: Apidae: Xylocopinae) presenti nelle collezioni entomologiche italiane: le collezioni della Sicilia. - Il Naturalista Campano 6: 1-5. 
